\title{
Monophosphoryl lipid A induces bone marrow precursor cells to differentiate into myeloid-derived suppressor cells
}

\author{
JIE CHEN ${ }^{1,2 *}$, BAOMU SUN ${ }^{3 *}$, XIUHUA ZHAO ${ }^{4 *}$, DONG LIANG $^{4}$, JUNXIA LIU $^{4}$, \\ YONG HUANG ${ }^{4}$, WANKE $\mathrm{LEI}^{5}, \mathrm{MAOMAO} \mathrm{CHEN}^{4}$ and WEIMIN SUN ${ }^{2}$ \\ ${ }^{1}$ Department of Ophthalmology, 117th Hospital of PLA, Hangzhou, Zhejiang 310013; \\ ${ }^{2}$ National Key Laboratory of Medical Immunology \& Institute of Immunology, Second Military Medical University; \\ ${ }^{3}$ Department of Integrated Chinese Traditional and Western Medicine, Eastern Hepatobiliary Surgical Hospital, \\ Second Military Medical University, Shanghai 200433; ${ }^{4}$ Department of Centre for Disease Prevention and Control, \\ Chengdu Military Region, Chengdu, Sichuan 610021; ${ }^{5}$ Department of Stomatology, The People's Hospital of Mianzhu, \\ Mianzhu, Sichuan 618200, P.R. China
}

Received April 8, 2013; Accepted July 18, 2013

DOI: $10.3892 / \mathrm{mmr} .2013 .1653$

\begin{abstract}
Myeloid-derived suppressor cells (MDSCs) and dendritic cells (DCs) are important in the immune response. In vitro, DCs are derived from myeloid precursors by stimulation with granulocyte macrophage colony-stimulating factor and interleukin-4. Previous studies demonstrated that lipopolysaccharide (LPS) in combination with interferon- $\gamma$ inhibited DC development but enhanced MDSC functions. Monophosphoryl lipid A (MPL), derived from LPS, is a unique immunomodulatory Toll-like receptor 4 agonist. In the present study, MPL was used to disturb DC differentiation from myeloid precursors and it was observed that prolonged stimulation with MPL led to the accumulation of MDSCs in vitro and in vivo. In conclusion, it was demonstrated that stimulation by MPL from the beginning of cell differentiation disturbed the development of DCs and led to the accumulation of MDSCs.
\end{abstract}

\section{Introduction}

Monophosphoryl lipid A (MPL) is a lipopolysaccharide (LPS)-derived Toll-like receptor 4 (TLR4) agonist that exhibits

Correspondence to: Dr Weimin Sun, Department of Immunology, National Key Laboratory of Medical Immunology \& Institute of Immunology, Second Military Medical University, 800 Xiangyin Road, Shanghai 200433, P.R. China

E-mail: sunchina@163.com

Dr Maomao Chen, Department of Centre for Disease Prevention and Control, Chengdu Military Region, 12 Tianxianqiao North Road, Chengdu, Sichuan 610021, P.R. China

E-mail: chenmaomao493@163.com

${ }^{*}$ Contributed equally

Key words: myeloid-derived suppressor cells, dendritic cells, monophosphoryl lipid A, myeloid precursor cells unique immunomodulatory properties at doses that are nonpyrogenic. In addition, it is a chemically detoxified lipid A moiety derived from Salmonella minnesota R595 LPS (1). Clinically, MPL is a component of several vaccine formulations. It has been demonstrated that MPL induces a strong phagocytic and a low inflammatory response via TLR4 (2).

Myeloid-derived suppressor cells (MDSCs) and dendritic cells (DCs) are derived from the same myeloid precursors; however, they exhibit different roles in the immune response. DCs demonstrated the capacity to initiate an innate and adaptive response $(3,4)$, while MDSCs have been observed to suppress immune responses via arginase, inducible nitric oxide synthase $(5,6)$, reactive oxygen species (7-14) and Foxp3 ${ }^{+}$regulatory cells $(15,16)$. The mechanisms that are involved in the differentiation of myeloid precursor cells into MDSCs instead of DCs have not been fully elucidated. It has been demonstrated that the development of DCs from bone marrow (BM) precursor cells in vitro is impaired in the presence of LPS (17). Another study showed that a combination of LPS and IFN- $\gamma$ inhibited DC development; however, enhanced the suppressive functions of MDSCs, including NO release and T cell suppression (18). In addition, other conserved structural patterns of microbial components, such as double-stranded RNA showed the capacity to regulate MDSC versus DC development from the same myeloid precursor (19).

As MPL is an active immunomodulator, in the present study, the involvement of MPL was investigated in the differentiation of myeloid precursor cells into MDSCs versus DCs. It was demonstrated in vivo and in vitro, that sustained stimulation with MPL inhibited the expansion of DCs and induced the development and expansion of MDSCs.

\section{Materials and methods}

Mice and treatments. Approximately 50 male and female wild-type C57BL/6 mice (age, 5-6 weeks) were purchased from the Chinese Academy of Sciences (Shanghai, China). DO11.10 OVA $323-339$-specific TCR-transgenic mice with a 
C57BL/6 background were obtained from The Jackson Laboratory (Bar Harbor, ME, USA). All mice were housed in a specific pathogen-free facility for all experiments. All animal procedures were undertaken in accordance with the National Institutes of Health Guide for the Care and Use of Laboratory Animals (NIH Publication No. 85-23, National Academy Press, Washington, DC, revised 1996), with the approval of the Laboratory Animal Center and Ethics Committee of the Second Military Medical University (Shanghai, China).

Reagents. Recombinant mouse granulocyte macrophage colony-stimulating factor (GM-CSF), interleukin (IL)-4, and an enzyme-linked immunosorbent assay (ELISA) kit for murine IL-12, IL-6, tumor necrosis factor (TNF)- $\alpha$, IL-10 and transforming growth factor (TGF)- $\beta$ were purchased from $R \& D$ Systems (Minneapolis, MN, USA). Fluorescein-conjugated mAbs to CD4,CD11b, CD80, CD86, Ia, CD40, CD11c, CCR7 and isotype control were purchased from Santa Cruz Biotechnology, Inc. (Santa Cruz, CA, USA). Fluorescein-conjugated mAbs to Gr1 were obtained from eBioscience (San Diego, CA, USA). LPS, 7-Aminoactinomycin D (7-AAD) and bovine serum albumin (BSA) were purchased from Sigma-Aldrich (Carlsbad, CA, USA).

Preparation of MPL from LPS of the gram-negative bacteria Salmonella minnesota R595. The MPL was prepared by eliminating the core oligosaccharide, hydrolyzing 1-phosphate from the reducing end glucosamine and removing the acyl chain from the 3 ' position of the disaccharide $(2,20,21)$.

Preparation of DCs from mouse BM and DC pre-treatment. $\mathrm{BM}$ mononuclear cells were prepared from mouse femur BM suspensions by depletion of red blood cells. The cells were then cultured at a density of $2 \times 10^{6}$ cells $/ \mathrm{ml}$ in 6 -well plates in RPMI-1640 medium supplemented with $10 \%$ fetal calf serum (FCS), $10 \mathrm{ng} / \mathrm{ml}$ recombinant mouse GM-CSF and $1 \mathrm{ng} / \mathrm{ml}$ recombinant mouse IL-4. Nonadherent cells were gently washed out on day 4 of culture. On day 5 , the dendritic proliferating clusters were collected and purified by anti-CD11c microbeads as immature dendritic cells (imDCs). ImDCs were stimulated with LPS (100 $\mathrm{ng} / \mathrm{ml})$ for another 2 days and then collected as mature DCs (mDCs). These cells were cultured in 24-well plates using $1 \times 10^{6}$ cell/well. All groups were cultured with granulocyte macrophage colony-stimulating factor and IL-4 throughout. MPL $(25 \mu \mathrm{g} / \mathrm{ml})$ was administered to the long stimulation group on day 0 and to the short stimulation group on day 5. On day 6, all cells were collected separately for further analysis (22).

Analysis of DC phagocytic ability. The cells of all groups were incubated at $37^{\circ} \mathrm{C}$ for $4 \mathrm{~h}$ with fluorescein isothiocyanate (FITC)-conjugated OVA at a final concentration of $100 \mu \mathrm{g} / \mathrm{ml}$ in RPMI-1640 medium containing 10\% FCS, were washed twice with ice-cold phosphate-buffered saline (PBS; $\mathrm{pH} 7.2$ ), containing $0.1 \% \mathrm{NaN}_{3}$ and $0.5 \% \mathrm{BSA}$, and were resuspended in chilled PBS for immediate flow cytometry. Cells were incubated with OVA-FITC at $4^{\circ} \mathrm{C}$.

Assay for cytokines and NO. Cytokines in the supernatant of the DC system were assayed with ELISA kits. NO production was assayed by the measurement of the nitrite concentration with the Griess assay.

Assays for Ag-specific CD $4^{+} T$-cell response. For the assay of the Ag-specific CD4 ${ }^{+}$T-cell proliferation, splenic $\mathrm{CD}^{+}{ }^{+} \mathrm{T}$ cells

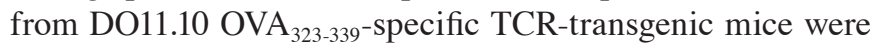
positively selected with anti-CD4-coated microbeads (Miltenyi Biotec, Bergisch Gladbach, Germany) by magnetic-activated cell sorting. The cells were cocultured with DCs treated as indicated in the presence of $\mathrm{OVA}_{323-339}$ peptide at a ratio of 1:10 (DC:T) in round-bottom 96 -well plates $\left(1 \times 10^{5} \mathrm{~T}\right.$ cells/200 $\mu \mathrm{l} /$ well $)$ for 5 days. The proliferation of the $\mathrm{T}$ cells was analyzed by double staining with anti-CD4 ${ }^{+}$and $7-\mathrm{AAD}^{-}$, and cells were counted by a fluorescence activated cell sorter (FACS).

Assay to determine the percentage of MDSCs and DCs in vivo. Six of the wild-type C57BL/6 mice were administered with MPL via the tail vein once (short stimulation) or once daily for 5 days (long stimulation). In total, there were 12 mice with 6 in the control group. The administration dosage was dependent upon the mouse body weight $(0.2 \mathrm{mg} / \mathrm{kg}$ or $2 \mathrm{mg} / \mathrm{kg})$. Following the preparation of single-cell suspensions from the mouse spleen, cells were stained with $\mathrm{Ab}-\mathrm{CD} 11 \mathrm{c}^{+}$conjugated FITC, Ab-CD11b+ conjugated R-Phycoerythrin (PE) or $\mathrm{Ab}-\mathrm{Grl}^{+}$conjugated FITC as indicated in the manufacturer's instructions.

Statistical analysis. Comparisons between experimental groups and relevant controls were performed by Student's t-test. $\mathrm{P}<0.05$ was considered to indicate a statistically significant difference.

\section{Results}

BM precursor cells exhibit a distinct phenotype and cytokine profile following long stimulation with MPL. Mouse BM-DCs were produced by standard protocol using GM-CSF and IL-4 (23-26). In vitro, the process of differentiation from myeloid precursor cells to DCs required 5 or 6 days. On day 0 , in the long stimulation group, myeloid precursor cells were isolated from mouse BM, washed and co-cultured with GM-CSF, IL-4 and MPL (20 $\mu \mathrm{g} / \mathrm{ml})$ for 5 days. In the short stimulation group, myeloid precursor cells were treated with GM-CSF and IL-4 only until day 5 . On day 5 , cells in the short stimulation group cells were treated once with MPL $(20 \mu \mathrm{g} / \mathrm{ml})$. On day 6 , cells from the two groups and the control group were harvested for further analysis. It was demonstrated that cells from the long stimulation group exhibited a lower expression of CD40, CD80, CD86 and Ia compared with that in the short stimulation group. Notably, CD11c expression in the cells of the long stimulation group was significantly decreased, which suggested that the development of DCs was blocked. Chemokine (C-C motif) receptor 7 (CCR7) was demonstrated to be responsible for directing the migration of DCs, in addition to the control of the cytoarchitecture, the rate of endocytosis, DC survival, migratory speed and DC maturation (27). In the present study, the expression of CCR7 was significantly decreased (Fig. 1A and B). Compared with the cytokine profile of the short stimulation and control groups, the cells in the long stimulation group secreted lower levels of IL-6, IL-12 and TNF- $\alpha$ and higher levels of IL-10 and NO (Fig. 1C). The cytokine profile of 
A

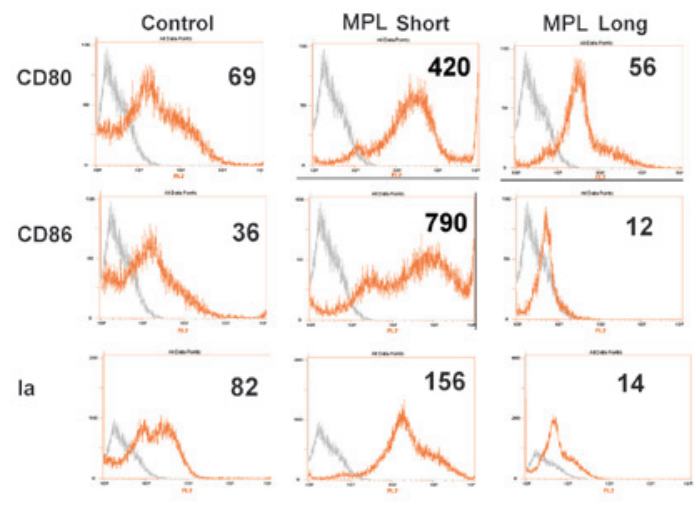

B
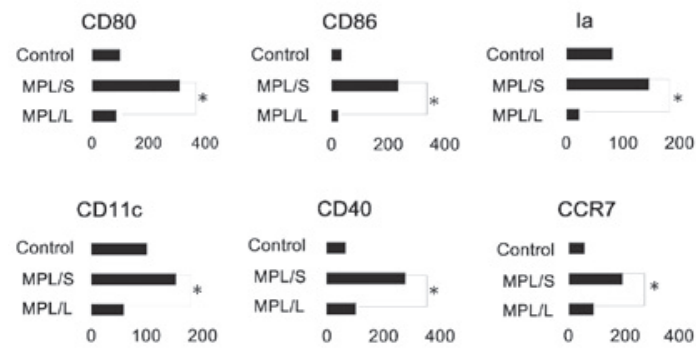

Mean Fluorescence Intensity (MFI)
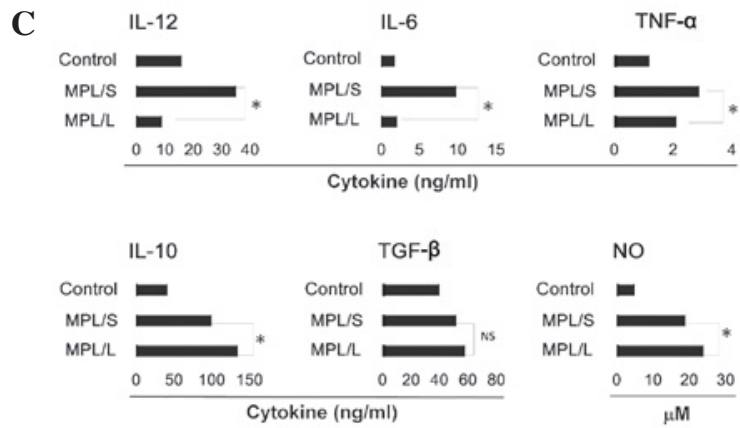

Figure 1. Phenotype and cytokine profiles of all groups. Myeloid precursor cells were generated from C57BL/6 mouse femur bone marrow suspensions by the depletion of red blood cells. These cells were cultured in 24-well plates using $1 \times 10^{6}$ cell/well. All groups were cultured with granulocyte macrophage colony-stimulating factor and IL-4 throughout. MPL was administered to the long stimulation group on day 0 and to the short stimulation group on day 5 . On day 6, all cells were collected separately for further analysis. (A) FACS charts of CD80, CD86 and Ia. Fold line in grey represents the blank control (cells without staining). Numbers in each chart represent the mean fluorescence intensity. (B) The expression of CD80, CD86, Ia, CD11c, CD40 and CCR7 was detected by FACS. Histograms represent the mean fluorescence intensity. Results are presented as the mean \pm SD of cells from triplicate wells. (C) NO expression and the cytokine profile of cells from different groups for $24 \mathrm{~h}$. On day 6 , cells of different groups were collected and washed in phosphate-buffered saline 3 times and placed in the wells for another $24 \mathrm{~h}$ IL-12, IL-6, tumor necrosis factor- $\alpha$, IL-10 and TGF- $\beta$ levels were assayed by an enzyme-linked immunosorbent assay and NO levels were assayed by a Griess assay. Results are presented as the mean \pm SD of triplicate wells ${ }^{*} \mathrm{P}<0.05$ compared with the short stimulation group. NS, no significance; IL, interleukin; MPL, monophosphoryl lipid A; FACS, fluorescence-activated cell sorting; NO, nitric oxide; TGF, transforming growth factor.

the long stimulation group suggested that the cells may exhibit an immunosuppressive capacity.

Cells from the long stimulation group show an enhanced phagocytic ability and the ability to suppress $T$ cell proliferation. The phenotype and cytokine profiles of cells from the MPL long stimulation group suggested that these cells may exhibit an immunosuppressive function. Thus, the cells were stained with $\mathrm{Grl}^{+} \mathrm{CD} 11 \mathrm{~b}^{+}$and analyzed by FACS. This demonstrated that double-positive $\mathrm{Grl}^{+} \mathrm{CD} 11 \mathrm{~b}^{+}$cells existed in the population (Fig. 2A and B). Therefore, it was hypothesized that these cells may be MDSCs. Subsequent to this, the phagocytic function of the different groups was analyzed, which demonstrated that cells from the long stimulation group exhibited a significantly increased phagocytic ability (Fig. 2C). In addition, the ability of DCs to stimulate antigen-specific T cell responses was investigated. It was observed that cells from the long stimulation group exhibited a reduced ability to induce the proliferation of OVA-specific $\mathrm{CD}^{+} \mathrm{T}$ cells. Notably, cells from the long stimulation group were added to the $\mathrm{mDCs} / \mathrm{CD} 4$ $\mathrm{T}$ cell coculture system and it was demonstrated that the T cell proliferation in vitro was partly suppressed (Fig. 2D).

MPL expands MDSC population and suppresses DC population in vivo. To investigate the effect of MPL in vivo, the MPL short and long stimulation model was conducted. Mice were administered with MPL via the tail vein once daily. In the short stimulation group, mice received MPL treatment on day 0 , then $24 \mathrm{~h}$ later, on day 1 , mice were euthanized. In the long stimulation group, mice received MPL treatment every $24 \mathrm{~h}$ and were euthanized on day 5 . The spleens were isolated from the two groups and the control to analyze the DC and MDSC population by FACS. It was demonstrated that MPL upregulated the percentage of DCs $\left(\mathrm{CD} 11 \mathrm{c}^{+}\right.$cells) and

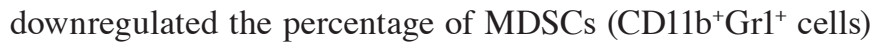
in the spleen. This data is consistent with the previous results (Fig. 3A and B).

\section{Discussion}

The results of the present study suggested that the TLR4 agonist (MPL) in the long stimulation group, disturbed the development of DCs and induced myeloid precursor cells to differentiate into $\mathrm{CD}_{11} \mathrm{~b}^{+} \mathrm{Grl}^{+}$cells (considered to be MDSCs) with an immunosuppressive function. Previous studies have demonstrated that factors that induce MDSC expansion include cyclooxygenase-2, prostaglandins (28-30), stem-cell factor (28), M-CSF, IL-6 (31), GM-CSF and vascular endothelial growth factor (32). In addition to expansion, the suppressive activity of MDSCs requires factors to induce their activation, which include IFN- $\gamma(33,34)$, ligands for Toll-like receptors, IL-13 (35), IL-4 and TGF- $\beta$ (35). In this study, IL-4, GM-CSF and MPL were investigated and were suggested to be involved in the differentiation of myeloid precursors into MDSCs.

MPL is a detoxified lipid A moiety derived from Salmonella minnesota R595 LPS. It is at least 100-fold less pyrogenic than LPS, yet maintains a number of the immunomodulatory properties of LPS (36). Previous studies have demonstrated that LPS and poly (I:C) may suppress the immune response $(18,19)$. The results indicated that MPL derived from LPS may also impair DC development.

The cells from the long stimulation group exhibited a $\mathrm{CD}_{11 b^{+}} \mathrm{Grl}^{+}$phenotype and were able to suppress $\mathrm{T}$ cell proliferation. This suggests that these cells exhibit an immunosuppressive function in the immune response. However, these 
A
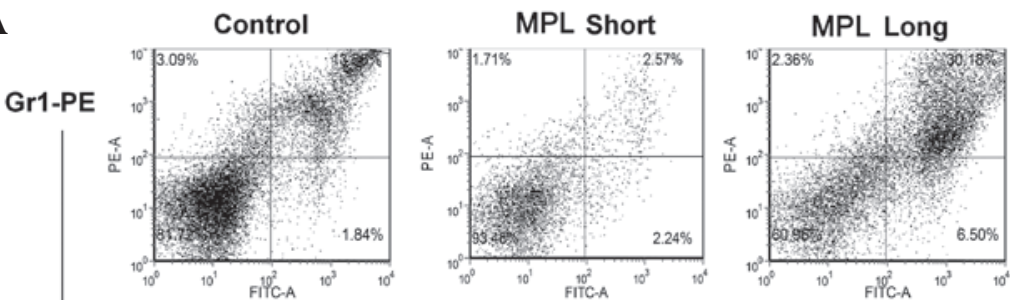

CD11b-FITC

B

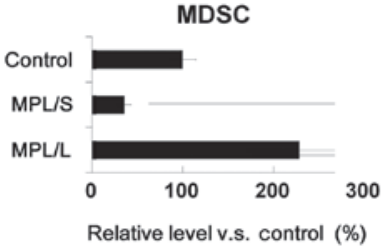

C

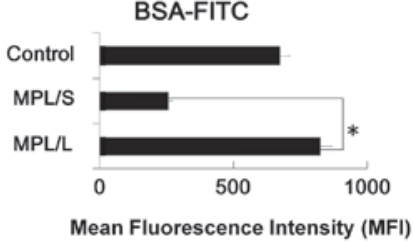

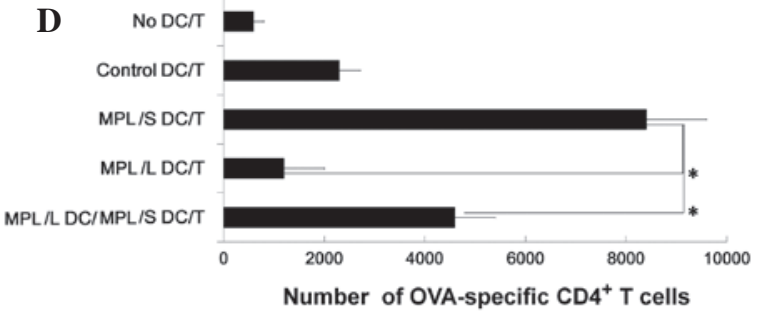

Figure 2. $\mathrm{Gr}^{+} \mathrm{CD} 11 \mathrm{~b}^{+}$cells in the long stimulation group showed an enhanced phagocytic ability and inhibited $\mathrm{CD}^{+} \mathrm{T}$ cell proliferation. (A) The phenotype of cells in the long stimulation group were analyzed by flow cytometry, the percentage of $\mathrm{Grl}^{+} \mathrm{CD} 11 \mathrm{~b}^{+}$cells was higher than in the other groups. (B) The percentage of $\mathrm{Grl}^{+} \mathrm{CD} 11 \mathrm{~b}^{+}$cells in all groups (the percentage in control group was referred as $100 \%$ ). Phagocytic ability was assessed for OVA-FITC phagocytosis by flow cytometry. Numbers in the histograms indicate the geometric mean fluorescence of test samples, cells $\left(1 \times 10^{6}\right.$ cell/well) were incubated with OVA-FITC at $4^{\circ} \mathrm{C}$ for $12 \mathrm{~h}$, washed with phosphate-buffered saline 3 times and analyzed by a fluorescence-activated cell sorter. (D) $\mathrm{CD}^{+} \mathrm{T}$ cells from DO11.10 OVA $\mathrm{O}_{323-339}$ specific (TCR-transgenic C57BL/6) F1 hybrid mice were cocultured with cells from all groups, 5 days later, the total number of viable $\mathrm{CD}^{+}{ }^{+} \mathrm{T}$ cells $\left(\mathrm{CD} 4{ }^{+}\right.$ $7 \mathrm{AAD}^{-}$) cells in each well was measured by flow cytometry. Results are presented as the mean \pm SD of three independent analyses. "P $<0.05$ compared with the short stimulation group. FITC, fluorescein isothiocyanate; 7-AAD, 7-aminoactinomycin D; MDSC, myeloid derived suppressor cell; MPL, monophosphoryl lipid A; DC, dendritic cell; BSA, bovine serum albumin.

A

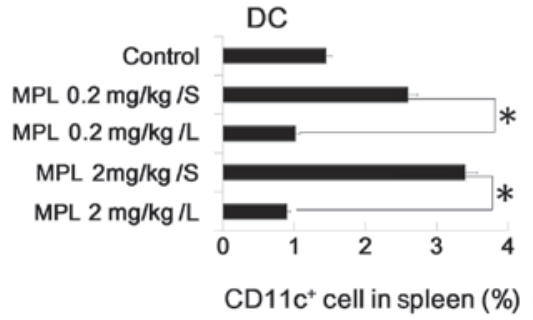

B

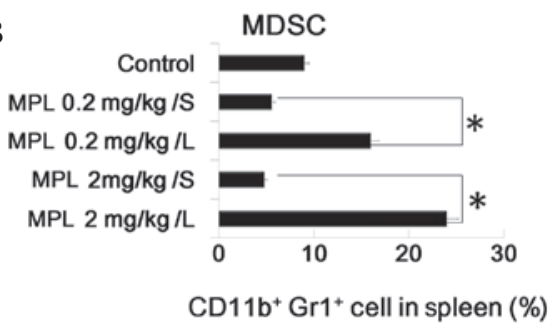

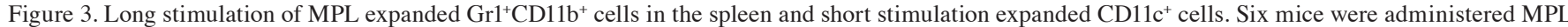
via the tail vein once a day for 1 day (short stimulation group) or for 5 days (long stimulation group). The dosage of administration was dependent upon mouse body weight $(0.2 \mathrm{mg} / \mathrm{kg}$ or $2 \mathrm{mg} / \mathrm{kg})$. Following the preparation of single-cell suspensions of spleen, cells were stained with Ab-CD11 ${ }^{+}$conjugated FITC, $\mathrm{Ab}-\mathrm{CD} 11 \mathrm{~b}^{+}$conjugated R-Phycoerythrin or Ab-Gr1 ${ }^{+}$conjugated FITC. These cells were then analyzed by a fluorescence-activated cell sorter. Results are presented as the mean \pm SD of three independent analyses. ${ }^{*} \mathrm{P}<0.05$ compared with the short stimulation group. (A) Percentage of $\mathrm{CD} 11 \mathrm{c}^{+}$cells in the spleen of all groups. (B) Percentage of $\mathrm{Grl}^{+} \mathrm{CD} 11 \mathrm{~b}^{+}$cells in the spleen of all groups. MPL, monophosphoryl lipid A; FITC, fluorescein isothiocyanate; MDSC, myeloid derived suppressor cell; DC, dendritic cell.

cells also showed enhanced phagocytic ability. A previous study demonstrated that stimulation of microglia and monocytes with MPL induced increased rates of phagocytosis of amyloid- $\beta(\mathrm{A} \beta)$ and that this mechanism may reduce the accumulation of $A \beta$ and improve spatial memory in APPswe/PS1 mice (2). Therefore, this similar phenomenon suggests that the enhanced phagocytic ability of the $\mathrm{CD}_{11} \mathrm{~b}^{+} \mathrm{Gr} 1^{+}$cells may have important roles in this process.
As BM precursor cells can be triggered to differentiate by conserved structural patterns of pathogens, it was questioned whetherDCs only have one fate. However, Abdi et al (37)demonstrated that LPS-activated DCs lose their responsiveness to LPS, yet remain capable of producing inflammatory cytokines in response to signals from activated T cells, CD40-ligand and soluble T cell-derived signals. Furthermore, these DCs retained sufficient plasticity to respond differentially to the interaction 
with Th0, Th1, Th2 and Th17 T cells (37). Thus, it appears that the MPL stimulated cells are not rested or exhausted, and suppress Th1 proliferation. However, the result of interaction with Th0, Th2 and Th17 cells and the understanding of whether these cells are equivalent to classical MDSCs remains to be elucidated.

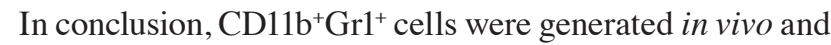
in vitro, and these cells showed an ability to suppress $\mathrm{CD} 4^{+}$ $\mathrm{T}$ cell proliferation and enhance phagocytic ability; however, further studies are required to fully determine this effect.

\section{Acknowledgements}

The authors would like to thank Dr Pu You and Dr Bing Yu from the Department of Cell Biology (Second Military Medical University, 800 Xiangyin Road, Shanghai 200433, P.R. China) for help with the discussion.

\section{References}

1. Casella CR and Mitchell TC: Putting endotoxin to work for us: monophosphoryl lipid A as a safe and effective vaccine adjuvant. Cell Mol Life Sci 65: 3231-3240, 2008.

2. Michaud JP, Hallé M, Lampron A, et al: Toll-like receptor 4 stimulation with the detoxified ligand monophosphoryl lipid A improves Alzheimer's disease-related pathology. Proc Natl Acad Sci USA 110: 1941-1946, 2013.

3. Steinman RM: The dendritic cell system and its role in immunogenicity. Annu Rev Immunol 9: 271-296, 1991.

4. Banchereau J and Steinman RM: Dendritic cells and the control of immunity. Nature 392: 245-252, 1998.

5. Bronte $\mathrm{V}$ and Zanovello P: Regulation of immune responses by L-arginine metabolism. Nat Rev Immunol 5: 641-654, 2005.

6. Rodríguez PC and Ochoa AC: Arginine regulation by myeloid derived suppressor cells and tolerance in cancer: mechanisms and therapeutic perspectives. Immunol Rev 222: 180-191, 2008.

7. Youn JI, Nagaraj S, Collazo M and Gabrilovich DI: Subsets of myeloid-derived suppressor cells in tumor-bearing mice. J Immunol 181: 5791-5802, 2008.

8. Kusmartsev S, Nefedova Y, Yoder D and Gabrilovich DI: Antigen-specific inhibition of $\mathrm{CD} 8^{+} \mathrm{T}$ cell response by immature myeloid cells in cancer is mediated by reactive oxygen species. J Immunol 172: 989-999, 2004.

9. Schmielau J and Finn OJ: Activated granulocytes and granulocyte-derived hydrogen peroxide are the underlying mechanism of suppression of t-cell function in advanced cancer patients. Cancer Res 61: 4756-4760, 2001.

10. Kusmartsev S, Nagaraj S and Gabrilovich DI: Tumor-associated $\mathrm{CD} 8^{+} \mathrm{T}$ cell tolerance induced by bone marrow-derived immature myeloid cells. J Immunol 175: 4583-4592, 2005.

11. Szuster-Ciesielska A, Hryciuk-Umer E, Stepulak A, Kupisz K and Kandefer-Szerszeń M: Reactive oxygen species production by blood neutrophils of patients with laryngeal carcinoma and antioxidative enzyme activity in their blood. Acta Oncol 43: 252-258, 2004

12. Waris G and Ahsan H: Reactive oxygen species: role in the development of cancer and various chronic conditions. J Carcinog 5: 14, 2006.

13. Mantovani G, Macciò A, Madeddu C, et al: Antioxidant agents are effective in inducing lymphocyte progression through cell cycle in advanced cancer patients: assessment of the most important laboratory indexes of cachexia and oxidative stress. J Mol Med (Berl) 81: 664-673, 2003.

14. Agostinelli E and Seiler N: Non-irradiation-derived reactive oxygen species (ROS) and cancer: therapeutic implications. Amino Acids 31: 341-355, 2006.

15. Yang R, Cai Z, Zhang Y, Yutzy WH, Roby KF and Roden RB: CD80 in immune suppression by mouse ovarian carcinoma-associated $\mathrm{Gr}-1^{+} \mathrm{CD} 11 \mathrm{~b}^{+}$myeloid cells. Cancer Res 66: 6807-6815, 2006

16. Huang B, Pan PY, Li Q, et al: Gr- $1^{+} \mathrm{CD} 115^{+}$immature myeloid suppressor cells mediate the development of tumor-induced $\mathrm{T}$ regulatory cells and T-cell anergy in tumor-bearing host. Cancer Res 66: 1123-1131, 2006.
17. Lutz MB, Kukutsch NA, Menges M, Rössner S and Schuler G: Culture of bone marrow cells in GM-CSF plus high doses of lipopolysaccharide generates exclusively immature dendritic cells which induce alloantigen-specific CD4 T cell anergy in vitro. Eur J Immunol 30: 1048-1052, 2000.

18. Greifenberg V, Ribechini E, Rössner S and Lutz MB: Myeloid-derived suppressor cell activation by combined LPS and IFN-gamma treatment impairs DC development. Eur J Immunol 39: 2865-2876, 2009.

19. Liu C, Zhang C, Lu H, et al: Poly(I:C) induce bone marrow precursor cells into myeloid-derived suppressor cells. Mol Cell Biochem 358: 317-323, 2011.

20. Baldridge JR and Crane RT: Monophosphoryl lipid A (MPL) formulations for the next generation of vaccines. Methods 19: 103-107, 1999.

21. Ulrich JT and Myers KR: Monophosphoryl lipid A as an adjuvant. Past experiences and new directions. Pharm Biotechnol 6: 495-524, 1995

22. Tsujimoto H, Efron PA, Matsumoto T, et al: Maturation of murine bone marrow-derived dendritic cells with poly(I:C) produces altered TLR-9 expression and response to CpG DNA. Immunol Lett 107: 155-162, 2006.

23. Zhang M, Tang H, Guo Z, et al: Splenic stroma drives mature dendritic cells to differentiate into regulatory dendritic cells. Nat Immunol 5: 1124-1133, 2004.

24. Tang H, Guo Z, Zhang M, Wang J, Chen G and Cao X: Endothelial stroma programs hematopoietic stem cells to differentiate into regulatory dendritic cells through IL-10. Blood 108: 1189-1197, 2006.

25. Xia S, Guo Z, Xu X, Yi H, Wang Q and Cao X: Hepatic microenvironment programs hematopoietic progenitor differentiation into regulatory dendritic cells, maintaining liver tolerance. Blood 112: 3175-3185, 2008.

26. Li Q, Guo Z, Xu X, Xia S and Cao X: Pulmonary stromal cells induce the generation of regulatory DC attenuating T-cell-mediated lung inflammation. Eur J Immunol 38: 2751-2761, 2008.

27. Sánchez-Sánchez N, Riol-Blanco L and Rodríguez-Fernández JL: The multiple personalities of the chemokine receptor CCR7 in dendritic cells. J Immunol 176: 5153-5159, 2006.

28. Pan PY, Wang GX, Yin B, et al: Reversion of immune tolerance in advanced malignancy: modulation of myeloid-derived suppressor cell development by blockade of stem-cell factor function. Blood 111: 219-228, 2008.

29. Sinha P, Clements VK, Fulton AM and Ostrand-Rosenberg S: Prostaglandin E2 promotes tumor progression by inducing myeloid-derived suppressor cells. Cancer Res 67: 4507-4513, 2007.

30. Serafini P, Carbley R, Noonan KA, Tan G, Bronte V and Borrello I: High-dose granulocyte-macrophage colony-stimulating factor-producing vaccines impair the immune response through the recruitment of myeloid suppressor cells. Cancer Res 64: 6337-6343, 2004.

31. Bunt SK, Yang L, Sinha P, Clements VK, Leips J and Ostrand-Rosenberg S: Reduced inflammation in the tumor microenvironment delays the accumulation of myeloid-derived suppressor cells and limits tumor progression. Cancer Res 67: 10019-10026, 2007.

32. Gabrilovich D, Ishida T, Oyama T, et al: Vascular endothelial growth factor inhibits the development of dendritic cells and dramatically affects the differentiation of multiple hematopoietic lineages in vivo. Blood 92: 4150-4166, 1998.

33. Kusmartsev S and Gabrilovich DI: STAT1 signaling regulates tumor-associated macrophage-mediated $\mathrm{T}$ cell deletion. J Immunol 174: 4880-4891, 2005.

34. Movahedi K, Guilliams M, Van den Bossche J, et al: Identification of discrete tumor-induced myeloid-derived suppressor cell subpopulations with distinct $\mathrm{T}$ cell-suppressive activity. Blood 111: 4233-4244, 2008.

35. Terabe M, Matsui S, Park JM, et al: Transforming growth factor-beta production and myeloid cells are an effector mechanism through which CD1d-restricted $\mathrm{T}$ cells block cytotoxic T lymphocyte-mediated tumor immunosurveillance: abrogation prevents tumor recurrence. J Exp Med 198: 1741-1752, 2003.

36. Cluff CW: Monophosphoryl lipid A (MPL) as an adjuvant for anti-cancer vaccines: clinical results. Adv Exp Med Biol 667: 111-123, 2010.

37. Abdi K, Singh NJ and Matzinger P: Lipopolysaccharide-activated dendritic cells: 'exhausted' or alert and waiting? J Immunol 188: 5981-5989, 2012. 